\title{
Conductivity and scattering in graphene bilayers: Numerically exact results versus Boltzmann approach
}

\author{
Hengyi Xu, T. Heinzel and Igor Zozoulenko
}

\section{Linköping University Post Print}

N.B.: When citing this work, cite the original article.

Original Publication:

Hengyi $\mathrm{Xu}, \mathrm{T}$. Heinzel and Igor Zozoulenko, Conductivity and scattering in graphene bilayers: Numerically exact results versus Boltzmann approach, 2011, Physical Review B. Condensed Matter and Materials Physics, (84), 11, 115409.

http://dx.doi.org/10.1103/PhysRevB.84.115409

Copyright: American Physical Society http://www.aps.org/

Postprint available at: Linköping University Electronic Press

http://urn.kb.se/resolve?urn=urn:nbn:se:liu:diva-71100 


\title{
Conductivity and scattering in graphene bilayers: Numerically exact results versus Boltzmann approach
}

\author{
Hengyi Xu and T. Heinzel \\ Condensed Matter Physics Laboratory, Heinrich-Heine-Universität, Universitätsstraße 1, D-40225 Düsseldorf, Germany \\ I. V. Zozoulenko* \\ Solid State Electronics, Department of Science and Technology (ITN), Linköping University, S-60174 Norrköping, Sweden
}

(Received 11 March 2011; published 12 September 2011)

\begin{abstract}
We derive analytical expressions for the conductivity of bilayer graphene (BLG) using the Boltzmann approach within the the Born approximation for a model of Gaussian disorders describing both short- and long-range impurity scattering. The range of validity of the Born approximation is established by comparing the analytical results to exact tight-binding numerical calculations. A comparison of the obtained density dependencies of the conductivity with experimental data shows that the BLG samples investigated experimentally so far are in the quantum scattering regime where the Fermi wavelength exceeds the effective impurity range. In this regime both short- and long-range scattering lead to the same linear density dependence of the conductivity. Our calculations imply that bilayer and single-layer graphene have the same scattering mechanisms. We also provide an upper limit for the effective, density-dependent spatial extension of the scatterers present in the experiments.
\end{abstract}

DOI: 10.1103/PhysRevB.84.115409

PACS number(s): 72.80.Vp, 72.10.Fk

\section{INTRODUCTION}

Single- and bilayer graphene have attracted tremendous attention since their discovery ${ }^{1}$ due to their fascinating and in many respects unique properties (for a review see, e.g., Refs. 2-4). Currently, one of the central issues in graphene research is to identify the scatterers that dominate the conductivity $\sigma$. This is not only a fundamental question, but also the prerequisite for progress regarding the quality of the electronic properties of graphene devices. Since the character of the scatterers manifests itself directly in the electron density $(n)$ dependence of $\sigma$, this function plays a key role in the corresponding experimental and theoretical investigations. ${ }^{3,4}$ Most experiments in single-layer graphene (SLG) show a dependence close to $\sigma \propto n,{ }^{4-7}$ while some submicrometer extra-clean suspended samples show a squareroot dependence $\sigma \propto n^{1 / 2}$ consistent with the ballistic transport regime. ${ }^{8-10}$ Within the standard Boltzmann transport theory in the Born approximation, ${ }^{11-13}$ the linear density dependence emerges from long-range Coulomb scattering which implies that charged impurities in the substrate are the dominant scatterers. Deviations from a linear dependence were attributed to effects of short-range impurities, ${ }^{5-7,14,15}$ for which the Boltzmann theory predicts $\sigma=$ const. $^{11-13}$ This point of view has been challenged by theories going beyond the first Born approximation ${ }^{16-18}$ as well as numerical simulations ${ }^{19,20}$ which show that short-range disorder leads to a linear density dependency similar to the one for the long-range potential. At the same time, growing evidence from recent experiments $^{21-23}$ points to short-range impurities as the main scattering mechanism in SLG.

The situation is much less clear in bilayer graphene (BLG). In most studies the BLG conductivity exhibits a linear density dependence very similar to that of SLG. ${ }^{7,22,24}$ A superlinear dependence was reported by Zhu et al., ${ }^{15}$ whereas a sublinear behavior has been observed in submicrometer BLG samples ${ }^{25}$ which may be an indication of quasiballistic transport. In the density regime where these experiments have been carried out, the energy dispersion of BLG is parabolic. For the case of short-range disorder, both the standard Boltzmann approach ${ }^{26,27}$ and theories not relying on the Born approximation ${ }^{28,29}$ predict $\sigma \propto n$. Recent numerical modeling of the BLG conductivity based on the Kubo approach shows that sufficiently far away from the neutrality point, a linear or sublinear dependence of $\sigma$ on $n$ is found, depending on the strength and type of the disorder. ${ }^{30,31}$ As far as the long-range disorder is concerned, the Boltzmann model in the Born approximation predicts $\sigma \propto n^{2}$ for unscreened, $\sigma \propto n$ for overscreened, and $\sigma \propto n^{\gamma}$ with $1<$ $\gamma<2$ for screened Coulomb disorder. ${ }^{27}$ These predictions are qualitatively different from the corresponding predictions for SLG. ${ }^{4,11-13}$ Therefore, in order to explain the observed density dependence (which is very similar for SLG and BLG), Das Sarma et $a l .{ }^{27}$ concluded that scattering mechanisms are fundamentally different for SGL and BLG, being dominated by the Coulomb impurities for the former and short-range impurities for the later. This is a rather surprising conclusion because scattering mechanisms in both systems are expected to be the same as both SLG and BLG are produced by the same manufacturing technique with the same substrate used in the measurements.

Since conclusions regarding the nature of the scattering mechanism in BLG are often based on the predictions of the standard Boltzmann approach, it is of the utmost importance to establish its range of validity and to study how well it models the exact conductivity. In the present study, we utilize the well-established model of Gaussian disorder, ${ }^{4,10,19,32-34}$ where the effective screening length $\xi$ can be chosen to describe both short- and long-range scattering, and derive analytical expressions for the conductivity of the BLG within the standard Boltzmann approach in the Born approximation. We then perform exact numerical tight-binding (TB) Landauer-type calculations and compare them with the analytical results. We demonstrate that for the density regime corresponding 
to the parabolic dispersion the exact TB calculations are consistent with the Boltzmann predictions. We show that in the experimentally relevant regime the latter predicts a linear density dependence of the conductivity for both short- and long-range scattering. This density dependence is in agreement with most experiments reported to date. Our calculations imply that the same scattering mechanisms dominate in BLG and SLG. We also provide an estimate for the range of effective screening lengths for the scatterers present in the experiments.

\section{DESCRIPTION OF THE MODEL}

We calculate $\sigma(n)$ of BLG using the standard $p$-orbital nearest-neighbor tight-binding Hamiltonian

$$
\begin{aligned}
H= & \sum_{\ell,\langle i, j\rangle}\left(V_{i} a_{\ell, i}^{+} a_{\ell, i}+V_{j} b_{\ell, j}^{+} b_{\ell, j}\right)-t \sum_{\ell,\langle i\rangle}\left(a_{\ell, i}^{+} b_{\ell, i+\Delta}+\text { H.c. }\right) \\
& -t_{\perp} \sum_{i}\left(a_{1, i}^{+} b_{2, i}+\text { H.c. }\right),
\end{aligned}
$$

with $V_{i}$ being the external potential at site $i, a_{\ell, i}^{+}\left(b_{\ell, j}^{+}\right)$being the creation operator at sublattice $A(B)$ and site $i(j)$ in layer $\ell=1,2$, and the coupling integrals $t=3.16 \mathrm{eV}, t_{\perp}=$ $0.39 \mathrm{eV}^{2-4}$ In the second term the index $i+\Delta$ corresponds to the nearest neighbors to the site $i$. In the parabolic dispersion regime we follow Yuan et al. ${ }^{30}$ and set $t_{\perp}=0.5 t$. This effectively extends the parabolic band to higher densities. Without this scaling and for the impurity concentrations used in our numerical calculations, the parabolic band would otherwise not be accessible since it is essentially replaced by the impurity band. Scattering centers are modeled by the Gaussian potential, ${ }^{4,10,19,32-34}$

$$
V\left(\mathbf{r}_{i}\right)=\sum_{i^{\prime}=1}^{N_{\text {imp }}} U_{i^{\prime}} \exp \left(-\frac{\left|\mathbf{r}_{i}-\mathbf{r}_{i^{\prime}}\right|^{2}}{2 \xi^{2}}\right) .
$$

We refer to the case $\xi=a$ (appropriate for the absorbed neutral impurities) as short-range scattering, and to the case $\xi \gg a$ (appropriate for the remote charged impurities) as long-range scattering, with $a$ being the $\mathrm{C}$-C distance. The potential heights are uniformly distributed in the range $U_{i} \in[-\delta, \delta]$ where $\delta$ denotes the maximum potential height. The correlator of this potential has also a Gaussian shape, ${ }^{32-34}$

$C(r) \equiv \sum_{i} V\left(\mathbf{r}_{i}\right) V\left(\mathbf{r}_{i}-\mathbf{r}\right)=\frac{K\left(\hbar v_{F}\right)^{2}}{2 \pi \xi^{2}} \exp \left(-\frac{r^{2}}{2 \xi^{2}}\right)$,

where the dimensionless impurity strength is described by the parameter $K \approx 40.5 n_{\text {imp }}(\delta / t)^{2}(\xi / \sqrt{3} a)^{4}$ with $n_{\text {imp }}$ being the relative concentration of impurities.

For the exact numerical calculations, we consider a rectangular BLG stripe of length $L$ and width $W$ exposed to the impurity potential according to Eq. (2) and attach it to semiinfinite leads. The conductivity of the stripe $\sigma(n)=\frac{L}{W} G(n)$ as a function of the electron density $n$ is obtained from the transmission coefficient $T$ which is related to the conductance $G$ via the Landauer formula $G=\frac{2 e^{2}}{h} T . T$ and $n$ are computed with the aid of the recursive Green's function technique. ${ }^{35,36}$ Because of computational limitations we study stripes with $L / W>1$. The obtained results are insensitive to $L / W$ as long as $L / W>1$.

\section{THE BOLTZMANN APPROACH}

Within the Boltzmann approach, the conductivity reads ${ }^{37}$

$$
\sigma=\frac{1}{2} e^{2} \tau D\left(E_{F}\right)\left(v_{F}^{B G}\right)^{2},
$$

where $D\left(E_{F}\right)$ is the density of states (DOS) at the Fermi energy, $v_{F}^{B G}$ is the Fermi velocity of the bilayer graphene, and $\tau$ is the scattering time. An analytical expression for the Boltzmann conductivity for the bilayer graphene can be obtained for the limiting cases of the parabolic and linear dispersions, $\hbar v_{F}|k| \ll \frac{t_{\perp}}{2}$ and $\hbar v_{F}|k| \gg \frac{t_{\perp}}{2}$, respectively [or, equivalently, $n \ll n_{0}$ and $n \gg n_{0}$, where $n_{0}=\frac{1}{\pi(3 a)^{2}}\left(\frac{t_{\perp}}{t}\right)^{2}$ is the critical density separating the parabolic and linear bands, and $v_{F}=\frac{3 a t}{2 \hbar}$ is the Fermi velocity of single-layer graphene].

In this section we present analytical expressions for the conductivity $\sigma$ for the bilayer graphene. The details of the derivation are given in the Appendix. For the parabolic dispersion $\left(n \ll n_{0}\right)$ the conductivity reads

$$
\begin{aligned}
\sigma & =\frac{e^{2} \hbar^{3}}{m^{* 2}} \frac{n}{K\left(\hbar v_{F}\right)^{2}} \frac{e^{z}}{\left(\frac{1}{z}-1\right) I_{1}(z)+\left(\frac{1}{z}+1\right) I_{2}(z)} \\
& \propto \begin{cases}n, & z \ll 1 \text { (quantum scattering), } \\
n^{5 / 2}, & z \gg 1 \text { (classical scattering), }\end{cases}
\end{aligned}
$$

where $I_{v}$ is the modified Bessel function, and $z=\pi n \xi^{2}=$ $\left(\frac{2 \pi \xi}{\lambda}\right)^{2}$ with $\lambda$ being the Fermi wavelength. It follows from the definition of $z$ that the condition $z \ll 1$ corresponds to the case of quantum scattering when the Fermi wavelength is larger than the effective width of the potential barrier, $\lambda \gg \xi$, while the opposite condition $z \gg 1$ corresponds to the case of classical scattering, $\lambda \ll \xi$.

For the linear dispersion $\left(n \gg n_{0}\right)$ the conductivity reads

$$
\begin{aligned}
\sigma & =\frac{16 e^{2}}{h} \frac{z e^{z}}{K\left(I_{1}(z)+I_{2}(z)\right)} \\
& \propto \begin{cases}\text { const, } & z \ll 1 \text { (quantum scattering) } \\
n^{3 / 2}, & z \gg 1 \text { (classical scattering) }\end{cases}
\end{aligned}
$$

Hence, it follows from Eqs. (5) and (6) that the Boltzmann approach for the Gaussian potential predicts four different regimes where the density dependence of the conductivity $\sigma=\sigma(n)$ is qualitatively different for parabolic and linear bands ( $n \ll n_{0}$ respectively $n \gg n_{0}$ ) and for quantum and classical scattering $(z \ll 1$ respectively $z \gg 1)$. The corresponding asymptotes for the density dependence for these four regimes are summarized in Table I.

TABLE I. Four different regimes for the density dependence for the conductivity of BLG as predicted by the Boltzmann theory, Eqs. (5), (6). It is noteworthy that the exponents for the linear band of BLG (regimes II and IV) are the same as those for SLG (Refs. 19 and 32).

\begin{tabular}{ccc}
\hline \hline & $\begin{array}{c}\text { Parabolic Band } \\
\left(n \ll n_{0}\right)\end{array}$ & $\begin{array}{c}\text { Linear Band } \\
\left(n \gg n_{0}\right)\end{array}$ \\
\hline Quantum scattering & Regime I: & Regime II: \\
$(z \ll 1)$ & $\sigma \propto n$ & $\sigma=$ const \\
Classical scattering & Regime III: & Regime IV: \\
$(z \gg 1)$ & $\sigma \propto n^{5 / 2}$ & $\sigma \propto n^{3 / 2}$ \\
\hline \hline
\end{tabular}




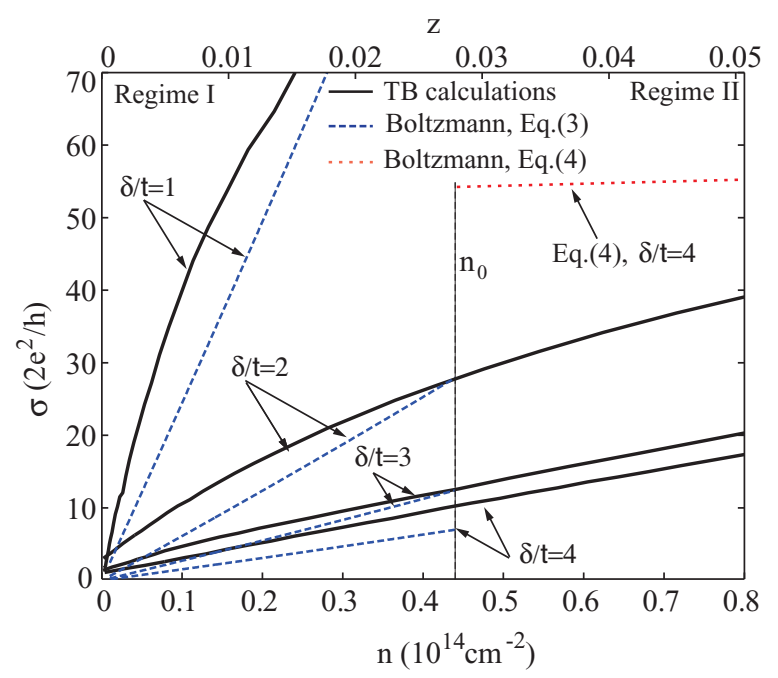

FIG. 1. (Color online) The density dependence of the TB and Boltzmann conductivities for Regime I (quantum scattering $z \ll 1$, parabolic band $n \ll n_{0}$ ) and Regime II (quantum scattering $z \ll 1$, linear band $\left.n \gg n_{0}\right) ; \xi=a ; t_{\perp}=0.5 t$, corresponding to $n_{0}=4.4 \times$ $10^{13} \mathrm{~cm}^{-2}$, for selected scattering strengths. The TB calculations are averaged over 1000 impurity configurations.

\section{EXACT RESULTS AND DISCUSSION}

As shown in the preceding section, for the case of the Gaussian potential the Boltzmann approach predicts four different regimes where the density dependence of the conductivity $\sigma=\sigma(n)$ is qualitatively different; see Eqs. (5), (6) and Table I. In the present section, we compare these predictions with the exact TB calculations.

Figure 1 displays the results for Regimes I and II (quantum scattering, $z \ll 1$, for the parabolic and linear band, respectively). Let us start with the most interesting Regime I of the parabolic band appropriate for most experiments (left part of the figure, $\left.n \ll n_{0}\right)$. The TB conductivity exhibits a density dependency close to the expected linear one, $\sigma \propto n$, and the TB and Boltzmann conductivities are in a reasonable quantitative agreement. Note that for smaller scattering strengths $(\delta \lesssim 2 t)$ the density dependence of $\sigma^{T B}$ is rather sublinear, which can be attributed to the quasiballistic transport regime (a transition from the ballistic to diffusive transport regimes in the SLG is discussed in Refs. 10 and 19). The calculations presented in Fig. 1 correspond to the short-range potential with $\xi=a$. Similar results are also obtained for a potential with a longer range, $\xi=3 a$ (not shown). Because of computational limitations we are not able to explore BLG conductivity in the Regime I for larger $\xi$. However we expect that for a given potential strength the agreement between the Boltzmann and the TB conductivities improves when the potential becomes smoother, i.e., when $\xi$ increases. A similar linear dependence of the BLG conductivity for the Gaussian potential in the parameter range $a \lesssim \xi \lesssim 5 a$ was also obtained by Yuan et al. ${ }^{30}$

Regime II of the quantum scattering case, $z \ll 1$, in the linear band corresponds to the right part of the figure, $n \gg n_{0}$. In this case the TB and Boltzmann conductivities disagree both qualitatively and quantitatively: The TB calculations show a linear dependence $\sigma^{T B} \propto n$, whereas the Boltzmann approach predicts $\sigma^{\text {Boltz }}=$ const. For the case of the linear band the BLG can be effectively regarded as two weakly interacting SLG sheets. Therefore the SLG conductivity and the conductivity of the BLG in the linear band are expected to show similar features. Indeed, the linear density dependence of the TB conductivity as well as a qualitative disagreement between the TB and Boltzmann approaches within the Born approximation for $z \ll 1$ have also been found for Gaussian scatterers for SLG. ${ }^{19}$

Why does the Born approximation for $z \ll 1$ work reasonably well (in the parameter region explored here) for the BLG in the parabolic band (Regime I), but fail for BLG in the linear band (Regime II) as well as for the SLG? The answer to this question can be obtained from an analysis of the validity of the Born approximation based on nonperturbative approaches. ${ }^{29,38}$ Using the $T$-matrix technique for the lattice model or the partial-wave expansion for the continuum description one can obtain an exact expression for the scattering rate for the case of the short-range ( $\delta$-function) impurities. The condition for the validity of the Born approximation for short-range scattering was discussed by Ferreira et al..$^{29,38}$ and reads

$$
\begin{gathered}
\frac{2}{\sqrt{\pi} 3^{3 / 4}}\left(V_{\text {eff }} / t\right) k R \ln (k R) \ll 1, \quad \text { SLG }, \\
\frac{18 t / t_{\perp}}{\left(V_{\text {eff }} / t\right)\left(c A_{1} / a^{2}\right)} \ll 1, \quad \text { BLG (parabolic band), }
\end{gathered}
$$

where $k$ is the Fermi wavelength, $R=\frac{3^{3 / 4}}{2 \sqrt{\pi}} a, A_{1}=\frac{3 \sqrt{3}}{4} a^{2}$ is the area per one $\mathrm{C}$ atom, $c$ is a constant of the order of 1 , and $V_{\text {eff }}$ is the effective impurity potential obtained using the $T$-matrix approach and the $a b$ initio calculations. ${ }^{20,39}$ For the SLG (and thus for the BLG in the linear band), the condition (7a) implies that the Born approximation is valid only for weak potentials, $V_{\text {eff }} / t \ll 5$. In contrast, condition (7b) is more relaxed, such that the Born approximation for the parabolic band of BLG is expected to work at least qualitatively even for strong realistic potentials, $V_{\text {eff }} / t \sim 60$, appropriate, e.g., for adsorbed hydrogen. ${ }^{20}$ In the present study, we present results for the short-range potential with $V_{\text {eff }} / t \lesssim 4$. For larger $V_{\text {eff }} / t$ the structure at hand for a given impurity concentration and system size enters the localization regime, where the Boltzmann approach is not applicable. Although we expect that the Born approximation would be qualitatively correct even for such strong potentials, it would be interesting to explore this case using numerical approaches capable of treating larger size structures, such as the Kubo approach. ${ }^{30}$

Let us now turn to the case of classical scattering, $z \gg 1$, corresponding to Regimes III and IV (parabolic and linear bands, respectively). We start by noting that for $z \gg 1$ the conductivity of SLG is well described by the Boltzmann approach within the Born approximation. ${ }^{19,32}$ We therefore expect a similar agreement between the Boltzmann and the TB calculations for BLG for the case of classical scattering in the linear band regime (i.e., in Regime IV). The conductivity of BLG in Regime IV (exemplified by choosing $\xi=16 a$ ) is shown in Fig. 2. According to the expectations, the TB calculations are in excellent qualitative agreement with the Boltzmann predictions showing the expected density dependence $\sigma \propto n^{3 / 2}$ and exhibiting the decrease of conductivity as the impurity strength $\delta$ is increased. However, the magnitude of the TB conductivity differs from its Boltzmann counterpart by a factor 3-6 with increasing deviation as $\delta$ increases. A similar 


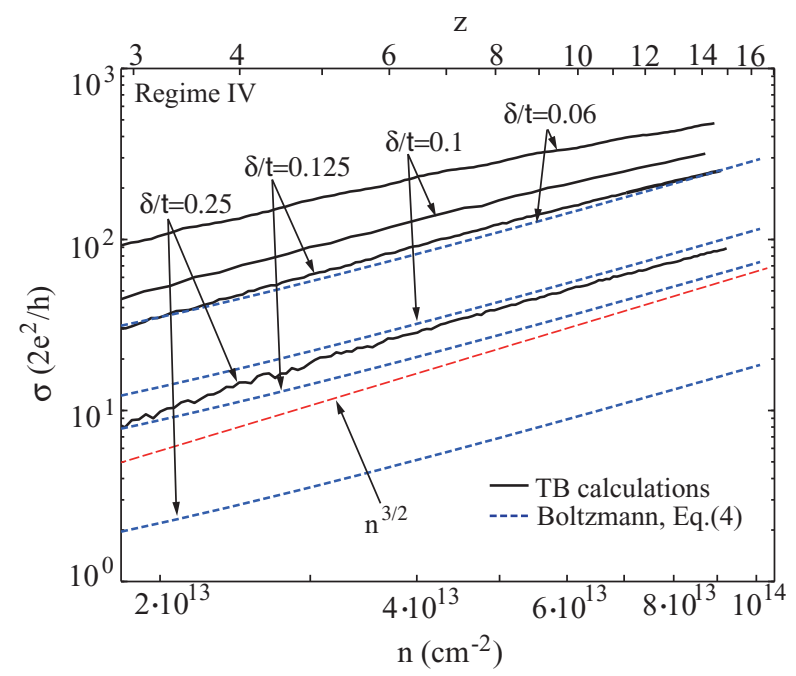

FIG. 2. (Color online) The density dependence of the TB and Boltzmann conductivities for Regime IV (classical scattering $z \gg 1$, linear band $\left.n \gg n_{0}\right) ; \xi=16 a ; t_{\perp}=0.39 \mathrm{eV}$, corresponding to $n_{0}=2.67 \times 10^{12} \mathrm{~cm}^{-2}$, for various scattering strengths. The TB calculations are averaged over 1000 impurity configurations. The sample size is $L \times W=320 \times 20 \mathrm{~nm}^{2}$.

quantitative discrepancy between the TB and the Boltzmann conductivities is also found for Regimes I and III. One of the reasons for this discrepancy is that due to computational limitations, the dimension of the structure $L$ is not sufficiently large to achieve a truly classical diffusive regime with

$$
L \gg l_{t r} \gg l_{F},
$$

where $l_{t r}=v \tau=\frac{h}{4 e^{2}} \frac{\sigma}{\sqrt{\pi n}}$ is the mean-free path and $l_{F}$ is the Fermi wavelength. For the scattering strengths $\delta$ used in calculations typical values of the mean-free path are $l_{t r} \simeq L / 2-L / 10(L=320 \mathrm{~nm}$ in Fig. 2 and $L=160 \mathrm{~nm}$ for Figs. 1 and 3 ) and $l_{F} \simeq 10 \mathrm{~nm}$. We expect that the agreement between the TB and Boltzmann conductivities would improve for larger structures where the condition Eq. (8) is better satisfied. Another reason for the discrepancy can be related to the utilization of the Born approximation, the applicability of which improves as the electron energy increases.

We finally turn to Regime III (classical scattering, $z \gg 1$, in the parabolic band). It is exemplified in Fig. 3 by choosing $\xi=32 a$. The TB calculations are in a qualitative agreement with the corresponding Boltzmann predictions showing the density dependence $\sigma \propto n^{5 / 2}$ expected for this regime and exhibiting a decrease of conductivity as the impurity strength $\delta$ is increased. We conclude this discussion by summarizing that for the classical scattering, $z \gg 1$, the TB calculations are consistent with the Boltzmann approach for both parabolic and linear bands, whereas for the quantum scattering, $z \ll 1$, the TB and Boltzmann calculations agree for the parabolic band and disagree for the linear band.

Let us relate our findings to available experimental data. Practically all the experiments on BLG structures reported so far have been performed for relatively low electron densities where the dispersion relation is parabolic. In most of these studies the BLG conductivity exhibits a linear density dependence. ${ }^{7,22,24}$ Out of four regimes considered above, this

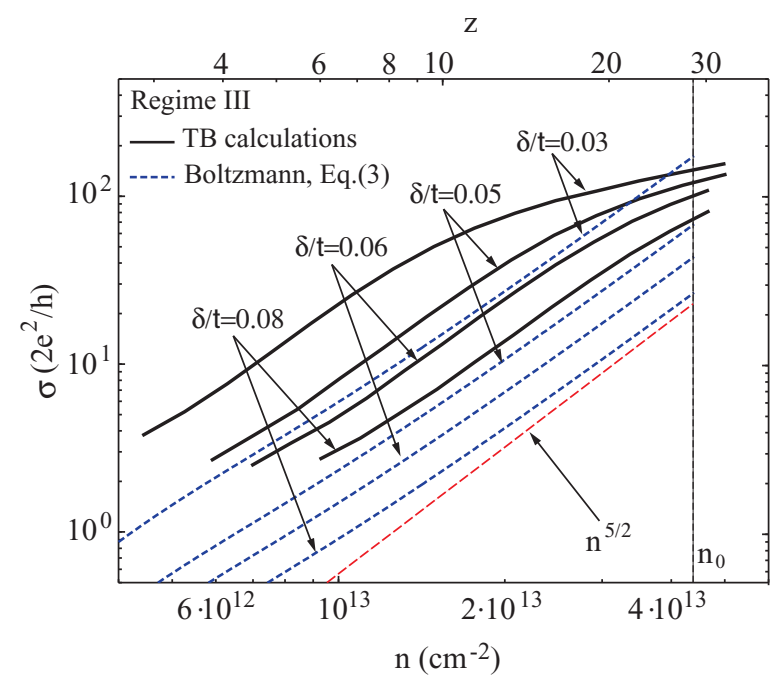

FIG. 3. (Color online) The density dependence of the TB and Boltzmann conductivities for Regime III (parabolic band, $n \ll n_{0}$ and weak scattering $z \gg 1$ ). $\xi=32 a ; t_{\perp}=0.5 t$ (corresponding to $n_{0}=4.4 \times 10^{13} \mathrm{~cm}^{-2}$ ), shown for different scattering strengths. The TB calculations are averaged over 1000 impurity configurations.

is consistent only with the Regime I (quantum scattering in the parabolic band) predicting a linear density dependence for both short- and long-range scatterers. We therefore conclude that Regime I is the regime corresponding to realistic BLG samples, and the experimentally observed linear dependence of the BLG conductivity can be caused by both short- and long-range scattering. Note that exact TB numerical calculations for SLG for the case of quantum scattering, $z \ll 1$, also give the same linear density dependence for both short- and long-range Gaussian scatterers. ${ }^{19}$ As the calculated and experimental conductivities of SLG and BLG are essentially the same, this strongly suggests that the scattering mechanisms for SLG and BLG cannot be different.

As far as the classical scattering $z \gg 1$ in the parabolic band is concerned (Regime II), the Boltzmann theory and the TB numerical calculations predict a superlinear density dependence $\sigma \propto n^{5 / 2}$. This dependence has never been observed in BLG structures. ${ }^{4}$ This leads us to conclude that the regime of the classical scattering, $z \gg 1$, is not accessible experimentally for BLG. (It is noteworthy that a similar conclusion, namely that the regime of classical scattering $z \gg 1$ is not achieved in SLG, was reported in Ref. 19). Therefore, the condition $z \equiv \pi n \xi^{2} \simeq 1$ provides an upper limit for the effective, density-dependent spatial extension of the scatterers present in the experiments. For example, for a typical electron density $n=10^{12} \mathrm{~cm}^{-2}$, we obtain $\xi \simeq 5.6 \mathrm{~nm}(\simeq 22$ lattice constants). This value of the effective screening length $\xi$ is consistent with the result reported by Ghaznavi et al ${ }^{40}$ for the Poisson-Thomas-Fermi screening of a charged impurity where a potential drops by a factor of 10 at distances $1-5 \mathrm{~nm}$.

\section{CONCLUSIONS}

We have studied the conductivity of BLG utilizing a model of Gaussian disorder where the effective screening length $\xi$ can be chosen to describe both short- and long-range scattering. 
Analytical expressions for the conductivity of BLG within the standard Boltzmann approach in the Born approximation have been derived and compared to exact numerical tightbinding Landauer-type calculations. Our main findings can be summarized as follows:

(1) The Boltzmann approach for the Gaussian potential predicts four different regimes where the density dependence of the conductivity $\sigma=\sigma(n)$ is qualitatively different for parabolic and linear bands $\left(n \ll n_{0}\right.$ respectively $\left.n \gg n_{0}\right)$ and for the quantum and classical scattering $(z \ll 1$ respectively $z \gg 1)$; see Table I for the summary. For the classical scattering, $z \gg 1$, the TB calculations are consistent with the Boltzmann approach for both parabolic and linear bands, whereas for the quantum scattering, $z \ll 1$, the TB and Boltzmann calculations agree for the parabolic band and disagree for the linear band. We discuss and analyze the applicability of the Born approximation for BLG and compare it with the case of SLG.

(2) By comparing the obtained density dependencies of the conductivity with available experimental results we conclude that realistic BLG samples are in the regime of the parabolic band, $n \ll n_{0}$, and of quantum scattering, $z \ll 1$. Here, both short- and long-range scattering lead to the same linear density dependence of the conductivity. We also compare the conductivities of SLG and BLG and conclude that they have the same scattering mechanisms.

(3) An upper limit for the effective, density-dependent spatial extension of the scatterers present in the experiments is provided. For example, for a typical electron density $n=$ $10^{12} \mathrm{~cm}^{-2}$, we obtain $\xi=5.6 \mathrm{~nm}$ (corresponding to 22 lattice constants).

\section{ACKNOWLEDGMENTS}

We are greatly thankful to N. M. R. Peres and A. Ferreira for illuminating discussions and correspondence and for sending us Ref. 29 prior to publication. We acknowledge communication with $\mathrm{S}$. Adam at the early stages of this work. I.V.Z. acknowledges the support of V.D. and V.R.

\section{APPENDIX : CALCULATION OF THE BOLTZMANN CONDUCTIVITY $\sigma$ FOR BILAYER GRAPHENE}

In this appendix we present a derivation of the Boltzmann conductivity for bilayer graphene for the case of the Gaussian potential. According to Eq. (4), calculations of the conductivity $\sigma$ require knowledge of the scattering time $\tau$, the DOS at the Fermi energy $D\left(E_{F}\right)$, and the Fermi velocity $v_{F}^{B G}$ of the bilayer graphene.

\section{A. Basics of bilayer graphene}

In this section we briefly summarize basic properties of bilayer graphene including the dispersion relations, wave functions, DOS, and the Fermi velocity that will be subsequently used below to calculate the conductivity $\sigma$ and the scattering time $\tau$.

We write the four-component wave function of the bilayer graphene in the form $\psi=\left(c^{A_{1}}, c^{B_{1}}, c^{A_{2}}, c^{B_{2}}\right)^{T} e^{i \mathbf{Q r}}$ where $A$ and $B$ correspond to the $A$ and $B$ sublattices, and 1 and 2 to layers, and $\mathbf{Q}$ is the wave vector. ${ }^{2,3}$ Substituting this wave function into the Schrödinger equation with the Hamiltonian Eq. (1) and expanding $\mathbf{Q}$ in the vicinity of the $\mathbf{K}$ point, $\mathbf{Q}=\mathbf{K}+\mathbf{k}$, we obtain

$$
\left(\begin{array}{lccc}
0 & f(\mathbf{q}) & \tilde{t}_{\perp} & 0 \\
f^{*}(\mathbf{q}) & 0 & 0 & 0 \\
\tilde{t}_{\perp} & 0 & 0 & f^{*}(\mathbf{q}) \\
0 & 0 & f(\mathbf{q}) & 0
\end{array}\right)\left(\begin{array}{l}
c^{A_{1}} \\
c^{B_{1}} \\
c^{A_{2}} \\
c^{B_{2}}
\end{array}\right)=-\widetilde{E}\left(\begin{array}{l}
c^{A_{1}} \\
c^{B_{1}} \\
c^{A_{2}} \\
c^{B_{2}}
\end{array}\right)
$$

where $\widetilde{E}=E / t, \widetilde{t}_{\perp}=t_{\perp} / t$, and

$$
\begin{aligned}
& f_{\mathbf{k}}=-\frac{3 a}{2}\left(k_{x}-i k_{y}\right)=-\left|f_{\mathbf{k}}\right| e^{-i \theta_{k}}, \\
& \left|f_{\mathbf{k}}\right|=\frac{3 a}{2} k=\frac{\hbar v_{F} k}{t}, \quad \theta_{k}=\tan \frac{k_{y}}{k_{x}} .
\end{aligned}
$$

The eigenvalues of Eq. (A1) are easily obtained,

$$
\widetilde{E}_{\mathbf{k}}=s_{1}\left(s_{2} \frac{\tilde{t}_{\perp}}{2}+\sqrt{\frac{\tilde{t}_{\perp}^{2}}{4}+\left|f_{\mathbf{k}}\right|^{2}}\right), \quad s_{1}, s_{2}= \pm 1,
$$

with the corresponding eigenfunctions

$$
\psi_{\mathbf{k}}(r)=\frac{1}{\sqrt{C}}\left(\begin{array}{c}
-\widetilde{E}_{\mathbf{k}} \\
f_{\mathbf{k}}^{*} \\
s_{1} s_{2} \widetilde{E}_{\mathbf{k}} \\
-s_{1} s_{2} f_{\mathbf{k}}
\end{array}\right) e^{i \mathbf{k r}},
$$

where the normalization constant $C=2\left(\widetilde{E}_{\mathbf{k}}^{2}+\left|f_{\mathbf{k}}\right|^{2}\right)$. The dispersion relation Eq. (A3) is illustrated in Fig. 4.

It is instrumental to consider two limiting cases where the dispersion relation Eq. (A3) shows qualitatively different behavior, (a) low energies, $\left|f_{\mathbf{k}}\right|<\frac{1}{2} \widetilde{t}_{\perp}\left(\hbar v_{F}|k|<\frac{1}{2} t_{\perp}\right)$ and (b) high energies, $\left|f_{\mathbf{k}}\right|<\frac{1}{2} \widetilde{t}_{\perp}\left(\hbar v_{F}|k|>\frac{1}{2} t_{\perp}\right)$. Using the relation between the wave number and the electron density in two dimensional (2D) electron systems, $k=\sqrt{\pi n}$, the conditions (a) and (b) can be rewritten in terms of the electron density as follows: (a) low densities, $n \ll n_{0}$, and (b) high densities, $n \gg n_{0}$, where $n_{0}=\frac{1}{\pi(3 a)^{2}}\left(t_{\perp} / t\right)^{2}$ is the

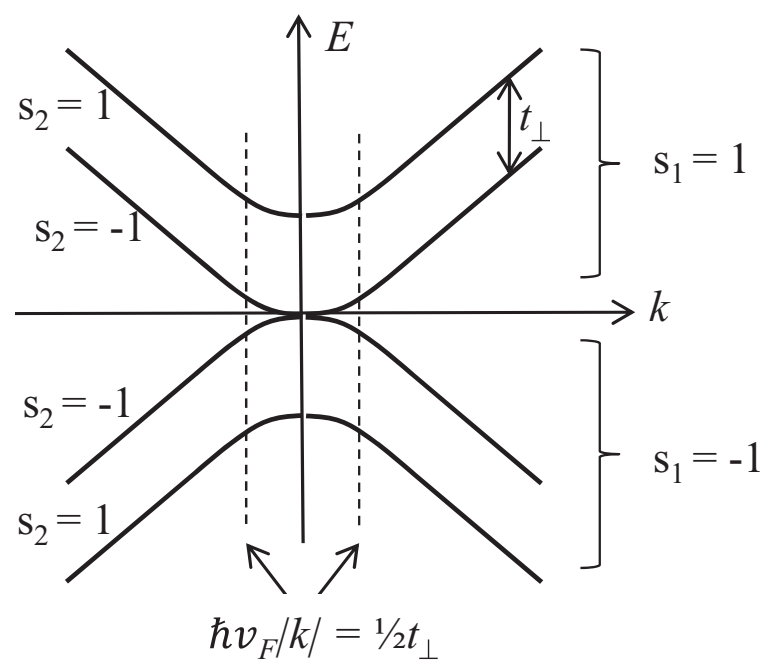

FIG. 4. A schematic illustration of the dispersion relation for a bilayer graphene, Eq. (A3). 
critical density separating the parabolic and linear bands. We will consider only the conduction band $\left(s_{1}=1\right)$; the same expressions for the conductivity can be obtained for the valence band $\left(s_{1}=-1\right)$.

\section{Low densities, $n \ll n_{0}$ (parabolic band)}

Introducing the effective mass $m^{*} \equiv \frac{t_{\perp}}{2 v_{F}^{2}}$, the dispersion relation Eq. (A3) for the lowest band $\left(s_{2}=-1\right)$ can be rewritten in a form familiar for conventional semiconductor systems,

$$
E=\frac{\hbar^{2} k^{2}}{2 m^{* 2}} .
$$

Hence, the Fermi velocity for the bilayer graphene $v_{F}^{B G}$ and its DOS have the same form as the ones for conventional 2D electron systems,

$$
v_{F}^{B G}=\hbar k / m^{*}, \quad D^{B G}(E)=\frac{g_{v} g_{s} m^{*}}{2 \pi \hbar^{2}}=\frac{2 m^{*}}{\pi \hbar^{2}},
$$

where the factors $g_{v}=g_{s}=2$ account for the valley and the spin degeneracy.

\section{High densities, $n \gg n_{0}$ (linear bands)}

For the high densities the dispersion relation consists of two linear bands (as in the case of the the single-layer graphene) separated by the energy interval $t_{\perp}$,

$$
E \approx\left\{\begin{array}{lc}
\hbar v_{F}|k|, & s_{2}=-1, \\
\hbar v_{F}|k|+t_{\perp}, & s_{2}=1 .
\end{array}\right.
$$

Each of these bands has the same dispersion as the one of single-layer graphene (SLG); therefore, in this case $v_{F}^{B G}=v_{F}$, and the DOS reads

$$
D^{B G}(E)=2 D^{S L G}(E)=\frac{g_{v} g_{s} E}{\pi\left(\hbar^{2} v_{F}^{2}\right)}=\frac{4 E}{\pi\left(\hbar^{2} v_{F}^{2}\right)},
$$

where a factor of two accounts for two bands.

\section{B. Calculation of the scattering rate}

Within the Boltzmann approximation the scattering rate reads $^{37}$

$$
\frac{1}{\tau}=\int \frac{d \mathbf{k}^{\prime}}{(2 \pi)^{2}} W_{\mathbf{k}, \mathbf{k}^{\prime}}(1-\cos \theta),
$$

where in the Born approximation $W_{\mathbf{k}, \mathbf{k}^{\prime}}$ is given by Fermi's golden rule,

$$
W_{\mathbf{k}, \mathbf{k}^{\prime}}=\frac{2 \pi}{\hbar}\left|V_{\mathbf{k}, \mathbf{k}^{\prime}}\right|^{2} \delta\left(E_{k}-E_{k^{\prime}}\right),
$$

with $\theta$ being the angle between the wave vectors $\mathbf{k}$ and $\mathbf{k}^{\prime}$, and $V_{\mathbf{k}, \mathbf{k}^{\prime}}$ is the matrix element of the scattering potential $V(\mathbf{r})$,

$$
V_{\mathbf{k}, \mathbf{k}^{\prime}}=\int \psi_{\mathbf{k}}^{\dagger} V(\mathbf{r}) \psi_{\mathbf{k}^{\prime}} d \mathbf{r} .
$$

Using the wave function Eq. (A4) we obtain for the matrix element

$$
V_{\mathbf{k}, \mathbf{k}^{\prime}}=\frac{2\left(\widetilde{E}_{k} \widetilde{E}_{k^{\prime}}+f_{k} f_{k^{\prime}}^{*}+f_{k}^{*} f_{k^{\prime}}\right)}{2 \sqrt{\widetilde{E}_{k}^{2}+\left|f_{k}\right|^{2}} \sqrt{\widetilde{E}_{k^{\prime}}^{2}+\left|f_{k^{\prime}}\right|^{2}}} U_{q},
$$

where $U_{q}=\int V(\mathbf{r}) e^{i \mathbf{q r}} d \mathbf{r}$ is the Fourier transform of the scattering potential, and $q=\left|\mathbf{k}-\mathbf{k}^{\prime}\right|=2 k \sin \frac{\theta}{2}$. We calculate $\left|U_{q}\right|^{2}$ making use of the Wiener-Khinchin theorem ${ }^{41}$ stating that the square modulus of the Fourier transform of a function is equal to the Fourier transform of its correlator, $\left|U_{q}\right|^{2}=$ $\int e^{i \mathbf{q r}} C(\mathbf{r}) d \mathbf{r}$. Using the expression Eq. (3) for the correlator of the Gaussian potential, we get

$$
\left|U_{q}\right|^{2}=K\left(\hbar v_{F}\right)^{2} e^{-\left(q^{2} \xi^{2} / 2\right)} .
$$

One can obtain analytical expressions for the scattering rate Eq. (A9) for two limiting cases of the low densities $(n \ll$ $\left.n_{0}\right)$ and the high densities $\left(n \gg n_{0}\right)$ where electrons obey respectively quadratic and linear dispersion relations.

\section{Low densities, $n \ll n_{0}$ (parabolic band)}

Using Eqs. (A2) and (A5) in Eq. (A12) we obtain

$$
\left|V_{\mathbf{k}, \mathbf{k}^{\prime}}\right|^{2}=\frac{1+\cos 2 \theta}{2}\left|U_{q}\right|^{2} .
$$

The expression for the scattering rate Eq. (A9) reads

$$
\frac{1}{\tau}=\frac{D^{B G}(E)}{4 \hbar} \int_{0}^{\pi} d \theta(1+\cos 2 \theta)(1-\cos \theta)\left|U_{q}\right|^{2},
$$

where we used the expression Eq. (A6) for the DOS. The Fourier transform of the Gaussian potential is given by the expression Eq. (A13). Substituting it into Eq. (A15) and performing integration utilizing that $q=2 k \sin \frac{\theta}{2}$, we obtain for the scattering rate

$$
\frac{1}{\tau}=\frac{\pi D(E)}{2 \hbar} K\left(\hbar v_{F}\right)^{2} e^{-z}\left[\left(\frac{1}{z}-1\right) I_{1}(z)+\left(\frac{1}{z}+1\right) I_{2}(z)\right],
$$

where $z=k^{2} \xi^{2}=\pi n \xi^{2}$, and $I_{v}(z)$ are the modified Bessel functions of the order $v$. Substituting this expression into Eq. (4) and using the definition of the DOS and $v_{F}^{B G}$ for the bilayer graphene, Eq. (A6), we finally obtain Eq. (5) for the Boltzmann conductivity.

\section{High densities, $n \gg n_{0}$ (linear bands)}

Using Eqs. (A2) and (A7) in Eq. (A12) we obtain

$$
\left|V_{\mathbf{k}, \mathbf{k}^{\prime}}\right|^{2}=\frac{(1+\cos \theta)^{2}}{4}\left|U_{q}\right|^{2} \text {. }
$$

Substituting the expression for into Eq. (A9) and using Eq. (A8), we obtain for the scattering rate

$$
\frac{1}{\tau}=\frac{D^{B G}}{16 \hbar} \int_{0}^{\pi} d \theta(1+\cos \theta)^{2}(1-\cos \theta)\left|U_{q}\right|^{2} .
$$

Substituting Eq. (A13) into this equation and performing integration using that $q=2 k \sin \frac{\theta}{2}$, we obtain for the scattering rate

$$
\frac{1}{\tau}=\frac{\pi D^{B G}}{16 \hbar} K\left(\hbar v_{F}\right)^{2} e^{-z}\left(\frac{I_{1}(z)+I_{2}(z)}{z}\right) .
$$

Substituting this expression into Eq. (4) and using the definition of the DOS and $v_{F}^{B G}$ for the bilayer graphene, Eq. (A8), we finally obtain Eq. (6) for the Boltzmann conductivity. 
*igor.zozoulenko@itn.liu.se

${ }^{1}$ K. S. Novoselov, A. K. Geim, S. V. Morozov, D. Jiang, Y. Zhang, S. V. Dubonos, I. V. Grigorieva, and A. A. Firsov, Science 306, 666 (2004).

${ }^{2}$ A. H. Castro Neto, F. Guinea, N. M. R. Peres, K. S. Novoselov, and A. K. Geim, Rev. Mod. Phys. 81, 109 (2009).

${ }^{3}$ S. Das Sarma, S. Adam, E. H. Hwang, and E. Rossi, Rev. Mod. Phys. 83, 407 (2011).

${ }^{4}$ N. M. R. Peres, Rev. Mod. Phys. 82, 2673 (2010).

${ }^{5}$ K. S. Novoselov, A. K. Geim, S. V. Morozov, D. Jiang, M. I. Katsnelson, I. V. Grigorieva, S. V. Dubonos, and A. A. Firsov, Nature (London) 438, 197 (2005).

${ }^{6}$ Y.-W. Tan, Y. Zhang, K. Bolotin, Y. Zhao, S. Adam, E. H. Hwang, S. Das Sarma, H. L. Stormer, and P. Kim, Phys. Rev. Lett. 99, 246803 (2007).

${ }^{7}$ S. V. Morozov, K. S. Novoselov, M. I. Katsnelson, F. Schedin, D. C. Elias, J. A. Jaszczak, and A. K. Geim, Phys. Rev. Lett. 100, 016602 (2008).

${ }^{8}$ X. Du, I. Skachko, A. Barker, and E. Y. Andrei, Nature Nanotechology 3, 491 (2008).

${ }^{9}$ K. I. Bolotin, K. J. Sikes, J. Hone, H. L. Stormer, and P. Kim, Phys. Rev. Lett. 101, 096802 (2008).

${ }^{10}$ J. W. Klos, A. A. Shylau, I. V. Zozoulenko, Hengyi Xu, and T. Heinzel, Phys. Rev. B 80, 245432 (2009).

${ }^{11}$ T. Ando, J. Phys. Soc. Jpn. 75, 074716 (2006).

${ }^{12}$ K. Nomura and A. H. MacDonald, Phys. Rev. Lett. 96, 256602 (2006).

${ }^{13}$ E. H. Hwang, S. Adam, and S. Das Sarma, Phys. Rev. Lett. 98, 186806 (2007).

${ }^{14}$ C. Jang, S. Adam, J.-H. Chen, E. D. Williams, S. Das Sarma, and M. S. Fuhrer, Phys. Rev. Lett. 101, 146805 (2008).

${ }^{15}$ W. Zhu, V. Perebeinos, M. Freitag, and Ph. Avouris, Phys. Rev. B 80, 235402 (2009).

${ }^{16}$ T. Stauber, N. M. R. Peres, and F. Guinea, Phys. Rev. B 76, 205423 (2007).

${ }^{17}$ M. I. Katsnelson and K. S. Novoselov, Solid State Commun. 143, 3 (2007).

${ }^{18}$ P. M. Ostrovsky, I. V. Gornyi, and A. D. Mirlin, Phys. Rev. B 74, 235443 (2006).

${ }^{19}$ J. W. Klos and I. V. Zozoulenko, Phys. Rev. B 82, 081414 (2010).

${ }^{20}$ T. O. Wehling, S. Yuan, A. I. Lichtenstein, A. K. Geim, and M. I. Katsnelson, Phys. Rev. Lett. 105, 056802 (2010).
${ }^{21}$ L. A. Ponomarenko, R. Yang, T. M. Mohiuddin, M. I. Katsnelson, K. S. Novoselov, S. V. Morozov, A. A. Zhukov, F. Schedin, E. W. Hill, and A. K. Geim, Phys. Rev. Lett. 102, 206603 (2009).

${ }^{22}$ M. Monteverde, C. Ojeda Aristizabal, R. Weil, K. Bennaceur, M. Ferrier, S. Gueron, C. Glattli, H. Bouchiat, J. N. Fuchs, and D. L. Maslov, Phys. Rev. Lett. 104, 126801 (2010).

${ }^{23}$ Z. H. Ni, L. A. Ponomarenko, R. R. Nair, R. Yang, S. Anissimova, I. V. Grigorieva, F. Schedin, Z. X. Shen, E. H. Hill, K. S. Novoselov, and A. K. Geim, Nano Lett. 10, 3868 (2010).

${ }^{24}$ S. Xiao, J.-H. Chen, S. Adam, E. D. Williams, and M. S. Fuhrer, Phys. Rev. B 82, 041406 (2010).

${ }^{25}$ B. Feldman, J. Martin, and A. Yacoby, Nature Phys. 5, 889 (2009).

${ }^{26}$ S. Adam and S. Das Sarma, Phys. Rev. B 77, 115436 (2008).

${ }^{27}$ S. Das Sarma, E. H. Hwang, and E. Rossi, Phys. Rev. B 81, 161407(R) (2010).

${ }^{28}$ M. I. Katsnelson, Phys. Rev. B 76, 073411 (2007).

${ }^{29}$ A. Ferreira, J. Viana-Gomes, J. Nilsson, E. R. Mucciolo, N. M. R. Peres, A. H. Castro Neto, Phys. Rev. B 83, 165402 (2011).

${ }^{30}$ S. Yuan, H. De Raedt, and M. I. Katsnelson, Phys. Rev. B 82, 235409 (2010).

${ }^{31}$ M. Trushin, J. Kailasvuori, J. Schliemann, and A. H. MacDonald, Phys. Rev. B 82, 155308 (2010).

${ }^{32}$ S. Adam, P. W. Brouwer, and S. Das Sarma, Phys. Rev. B 79, 201404(R) (2009).

${ }^{33}$ J. H. Bardarson, J. Tworzydło, P. W. Brouwer, and C. W. J. Beenakker, Phys. Rev. Lett. 99, 106801 (2007).

${ }^{34}$ C. H. Lewenkopf, E. R. Mucciolo, and A. H. Castro Neto, Phys. Rev. B 77, 081410(R) (2008).

${ }^{35}$ H. Xu, T. Heinzel, and I. V. Zozoulenko, Phys. Rev. B 80, 045308 (2009).

${ }^{36} \mathrm{H}$. Xu, T. Heinzel, M. Evaldsson, and I. V. Zozoulenko, Phys. Rev. B 77, 245401 (2008).

${ }^{37}$ N. W. Ashcroft and N. D. Mermin, Solid State Physics (Saunders College Publishing, Philadelphia, 1976).

${ }^{38}$ A. Ferreira and N. M. R. Peres (private communication).

${ }^{39}$ J. P. Robinson, H. Schomerus, L. Oroszlány, and V. I. Fal'ko, Phys. Rev. Lett. 101, 196803 (2008).

${ }^{40}$ M. Ghaznavi, Z. L. Mišković, and F. O. Goodman, Phys. Rev. B 81, 085416 (2010).

${ }^{41}$ W. H. Press, S. A. Teukolsky, W. Vetterling, and Brian P. Flannery, Numerical Recipes: The Art of Scientific Computing (Cambridge University Press, Cambridge, 2007). 